midwife (PHM) area, Vidatalativu East PHM and Vidatalativu West PHM area. These areas were randomly selected PHM areas from the resettlement areas in Mannar. Sample size was selected as a $25 \%$ of the population. Simple random sampling method was done, house holds were randomised. Two members were invited from each house hold.

Results There are 87 participants from all three areas. 48 (55\%) were females. 15 (17\%) were children. In our study we found $79 \%(n=27$, $95 \%$ CI 11.6 to 12.7 , SD 1.58$)$ of men are anaemic and $60 \%(n=23$, $95 \%$ CI 11.0 to $12.0, \mathrm{SD} 1.4$ ) of women are anaemic. In children $53 \%$ $(\mathrm{n}=8,95 \%$ CI 10.6 to 12.3 , SD 1.54$)$ of them are anaemic.

Conclusions According to the previous studies in Sri Lanka this small study shows very high prevalence of anaemia among these resettled populations.

\section{SP6-5 INFLUENZA VACCINATION AMONG ELDERLY PERSONS: PREVALENCE, ASSOCIATED FACTORS AND REASONS FOR NON-COMPLIANCE IN CAMPINAS, STATE OF SÃO PAULO, BRAZIL}

doi:10.1136/jech.2011.142976p.76

P Francisco, ${ }^{*}$ M Barros, M R Cordeiro. University of Campinas, Campinas, São Paulo, Brazil

Introduction The benefits of influenza vaccination among the elderly and people with chronic conditions have been shown in several studies. The aim this study was to assess the influenza vaccination coverage in the elderly, identifying associated factors and reasons for non-compliance.

Methods This was a cross-sectional population-based study with stratified clustered two-stage sampling in Campinas, São Paulo, Brazil in 2008-2009.

Results Among the 1517 elderly, 62.6\% reported influenza vaccination during the previous year. Physical activity in leisure, selfreported diabetes and recommendation from a healthcare worker were positively associated with the vaccination, while occupational activity and hospitalisation were negatively statistically related. Except for diabetics, the vaccination didn't reach adequate levels in elderly with chronic diseases. Reasons for non-compliance were: not find it necessary and belief that the vaccine causes reaction.

Conclusion Socioeconomic conditions, life style and physical mobility did not restrict adherence to vaccination, but the coverage was below the target of $80 \%$ established by the Ministry of Health. Campaigns focused on the elderly aged 60-69 years and on patients with chronic conditions, beyond the guidance of health professionals can expand coverage to groups with low adherence to the campaigns.

\section{SP6-6 COMPARISON OF ESTIMATES FOR THE SELF-REPORTED CHRONIC CONDITIONS: ISACAMP AND VIGITEL-CAMPINAS}

doi:10.1136/jech.2011.142976p.77

${ }^{1} \mathrm{P}$ Francisco, ${ }^{*}{ }^{1} \mathrm{M}$ Barros, ${ }^{2} \mathrm{~N}$ Segri, ${ }^{3} \mathrm{C}$ Alves, ${ }^{2} \mathrm{C}$ Cesar, ${ }^{4} \mathrm{D}$ Malta. ${ }^{1}$ University of Campinas, Campinas, São Paulo, Brazil; '2University of São Paulo, São Paulo, São Paulo, Brazil; ${ }^{3}$ Institute of Health, São Paulo, São Paulo, Brazil; ${ }^{4}$ Ministry of Health, Brasilia, Distrito Federal, Brazil

Introduction Population surveys are tools widely used in epidemiological studies to produce information needed for formulating and evaluating social policies and interventions in health. The aim of this study was to compare the estimates obtained by different methods of population-based surveys for self-reported chronic conditions among adults living in Campinas in the year 2008.

Methods Data from ISACamp Survey, conducted by the Faculty of Medical Sciences from State University of Campinas (UNICAMP) with support from the Municipal Health Department and VIGITEL (Campinas), a telephone survey conducted by the Brazilian Ministry of Health towards Surveillance of Risk and Protection Factors for Chronic non-transmittable Diseases in the adult population (18 years and over) were analysed. Estimates of self-reported hypertension, diabetes, osteoporosis, asthma/bronchitis/emphysema were evaluated and compared using the test for two independent samples.

Results For the global estimates, the higher prevalence of hypertension and osteoporosis were ascertained by the telephone survey. Diabetes and asthma/bronchitis/emphysema showed no significant statistical differences. According to socio-demographic variables, a higher prevalence of hypertension was obtained by VIGITEL to men, aged 18-59 years old and who reported nine or more years of schooling. A higher prevalence of osteoporosis among adults (18-59 years) was verified by VIGITEL. Concerning to asthma/ bronchitis/emphysema in the elderly, a higher prevalence was observed by ISACamp survey.

Conclusion Except for hypertension, data from the telephone survey provided a rapid alternative to provide global prevalence estimates of health conditions in the adult population in Campinas.

\section{SP6-7 TIME TRENDS IN MORTALITY DUE TO MOTORCYCLE ACCIDENTS IN DISTRITO FEDERAL, BRAZIL, 1996-2007}

doi:10.1136/jech.2011.142976p.78

${ }^{1,2} \mathrm{~A}$ Nascimento, ${ }^{*} \mathrm{M}$ de Mesquita Silva Montenegro, ${ }^{2} \mathrm{~K}$ B Ribeiro. ${ }^{1}$ Institute of Education and Science, Hospital Alemão Oswaldo Cruz, São Paulo, São Paulo, Brazil; ${ }^{2}$ Department of Social Medicine, Faculdade de Ciências Médicas da Santa Casa de São Paulo, São Paulo, São Paulo, Brazil

Introduction Motorcycle accidents are increasing in several parts of world (Asia, Africa and Latin America) and are associated with high levels of mortality and morbidity.

Objectives To assess time trends in mortality due to motorcycle accidents in Distrito Federal, Brazil, in the period between 1996 and 2007.

Methods Mortality data and estimates of resident population, stratified by age and sex were obtained from the Brazilian Mortality Information System (SIM) for 1996-2007. Age-standardised mortality rates were calculated by the direct method using the 2000 Brazilian population. Trends were modelled using linear regression. Joinpoint regression method was used to study the annual per cent change in mortality rates.

Results There were 580 deaths of motorcyclists during the studied period. They were men (94.3\%), aged between 20 and 29-year-old $(73.8 \%)$ and mulattos $(71.0 \%)$. Standard mortality rate of motorcyclists among males was 1.9 death/100 000 males in 1996 and increased to 7.2 deaths/100 000 males in 2007. In 1998 there were 2.0 deaths $/ 10000$ motorcycles and in 2007 there were 10 deaths/10 000 motorcycles in DF. The estimated annual increase in age-standardised mortality rate was 0.48 deaths $/ 100000$ men (95\% CI 0.31 to $0.65 ; \mathrm{p}<0.001$ ). The annual per cent change in age-standardised mortality rate among males in the period between 1998 and 2007 was 36.2\% (95\% CI 21.2\% to 53.2\%; $\mathrm{p}<0.05)$.

Conclusions There was an important increase in mortality due to motorcycle accidents. The fleet explained in part this increase. But additional studies are necessary to investigate factors associated to this type of transport accidents. 